\title{
Fluids density functional theory studies of supramolecular polymers at a hard surface
}

\author{
E. S. McGarrity, ${ }^{1, a)}$ J. M. Thijssen, ${ }^{2}$ and N. A. M. Besseling ${ }^{3}$ \\ ${ }_{1}^{1}$ Engineering Thermodynamics Section, Process and Energy, Delft University of Technology, \\ 2628 CA Delft, The Netherlands \\ ${ }_{2}^{2}$ Kavli Institute of Nanotechnology, Delft University of Technology, 2628 BL Delft, The Netherlands \\ ${ }^{3}$ Department of Chemical Engineering, Nanostructured Materials Section, Delft University of Technology, \\ 2628 CJ Delft, The Netherlands
}

(Received 11 March 2010; accepted 1 July 2010; published online 26 August 2010)

\begin{abstract}
We have applied a fluids density functional theory based on that of $\mathrm{Yu}$ and $\mathrm{Wu}$ [J. Chem. Phys. 116, 7094 (2002)] to treat reversible supramolecular polymers near a hard surface. This approach combines a hard-sphere fluids density functional theory with the first-order thermodynamic perturbation theory of Wertheim. The supramolecular polymers are represented in the theory by hard-spheres with two associating sites. We explore the effects of the bonding scheme, monomer concentration, and association energy upon the equilibrium chain sizes and the depletion lengths. This study is performed on simple systems containing two-site monomers and binary mixtures of two-site monomers combined with end stopper monomers which have only a single association site. Our model has correct behavior in the dilute and overlap regimes and the bulk results can be easily connected to simpler random-flight models. We find that there is a nonmonotonic behavior of the depletion length of the polymers as a function of concentration and that this depletion length can be controlled through the concentration of end stoppers. These results are applicable to the study of colloidal dispersions in supramolecular polymer solutions. (C) 2010 American Institute of Physics. [doi:10.1063/1.3469789]
\end{abstract}

\section{INTRODUCTION}

Supramolecular polymers are composed of small molecules, often called monomers, which have reversible bonding sites. The reversible nature of the bonds formed in supramolecular chains makes their physical properties, such as molecular weight, behave quite differently from standard covalently bonded polymers. ${ }^{1}$ When placed in solution, supramolecular polymers spontaneously form and break bonds at rates determined by their density, bonding motif, and external factors such as solvent type, temperature, $\mathrm{pH}$, etc. These polymers have potential applications as so-called smart materials which can change their properties depending on their environmental conditions. ${ }^{2,3}$ For instance, such polymers have been used to control the stability of a colloidal suspension. ${ }^{4}$ Other examples of supramolecular polymers include liquid sulfur, ${ }^{5-7}$ wormlike micelles, ${ }^{8}$ and DNA tiling. ${ }^{9,10}$

There is a large body of literature on the theory of associating fluids which can be roughly divided into four categories. The first category is based on the Percus-Yevick theory for classical fluids. ${ }^{11-13}$ In that work, Cummings and Stell created a model for reversible chemical bonding and applied it to dimerizing fluids. Another approach is to treat the polymers with a path integral theory based on the model of Edwards. ${ }^{14,15}$ This theory has been adapted for supramolecular polymers, and in some cases the solution of the equations can be simplified considerably. ${ }^{16-20} \mathrm{~A}$ third method for deal-

${ }^{a)}$ Electronic mail: e.s.mcgarrity@tudelft.nl. ing with equilibrium polymers is based on the BetheGuggenheim lattice theory for polymers. ${ }^{21}$ There the polymer solution is treated as a lattice fluid composed of monomers with two binding sites each. Given an energy of association and a density, the partition function can be expressed in terms of lattice site occupations and binding site contact statistics. ${ }^{22-26}$ The final category of theories is based on extensions of Wertheim's thermodynamic perturbation theory for associating fluids. ${ }^{27-32}$ These extensions have been used to treat associating liquids in bulk and near interfaces. The treatment most relevant to the work herein is the statistical associating fluids theory (SAFT) of Chapman et al. ${ }^{33}$ For a review of SAFT see Müller and Gubbins. ${ }^{34}$ This theory was further extended into a fluids density functional theory (FDFT) and used successfully to treat systems with interfaces, such as dimers, polymer chains, ${ }^{35-40}$ polymer brushes, ${ }^{41,42}$ and water. ${ }^{43-46} \mathrm{~A}$ recent comprehensive review of FDFT contains many more examples of the successful uses of the theory. ${ }^{47}$

In addition to the theoretical work above, there have been several simulation studies of associating systems. Particularly, there have been investigations into bulk two and four site associating fluids, ${ }^{48-50}$ as well as those located near a wall. ${ }^{51}$ Further, there has also been some work done with simulations to study equilibrium chains near a surface ${ }^{52}$ and end-adsorbed systems which mimic polymer brushes. ${ }^{53,54}$

In this work we apply a hard-sphere FDFT with an association perturbation to an investigation of the physical properties of supramolecular polymers in the presence of a hard wall. These supramolecular polymers are formed from 


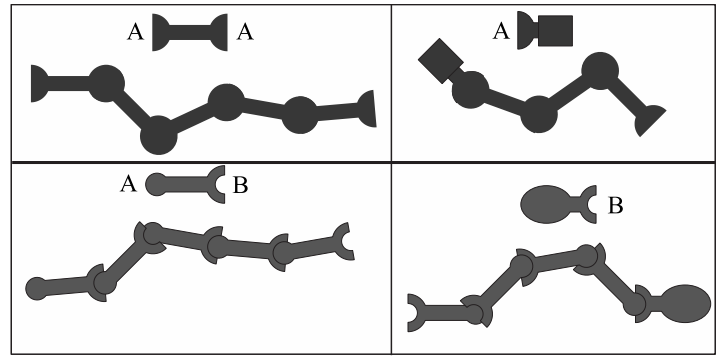

FIG. 1. Various bonding motifs exist for associating monomers with two bonding sites and stopper monomers. The top left motif has bonding sites with self-affinity. The bottom left only allows bonding between sites of types A and B. Adding stoppers which have affinity for one of the types of end groups results in the two right-hand schemas.

monomers which have two bonding sites. The effect of bonding site specificity is investigated and the results are expressed in the traditional language of polymers. In addition, these systems are compared to and validated against previous lattice-based mean-field calculations. ${ }^{22}$ We also present calculations of binary systems containing one species with two bonding sites and another species of so-called chainstoppers, which have only a single bonding patch. The behavior of these systems is shown to be governed mainly by the concentration of stoppers.

This paper is organized into three main sections. The model for the monomeric system and an overview of the FDFT are given in Sec. II. The calculation results and an analysis of how they fit within the language of polymers are presented in Sec. III. Finally, concluding remarks are given in Sec. IV.

\section{MODEL AND THEORY}

The model we employ for supramolecular polymers is shown schematically in Fig. 1. Depicted in this figure are several types of bonding motifs that we investigate. These can be placed into four categories based on the combination of unary/binary fluids with specific/nonspecific bonding sites. For single species systems with two bonding sites per monomer, there are two cases which depend on the binding groups present. In the first type, the monomers have two self-complimentary bonding sites. Polymers formed from this type of monomer have no particular orientation along their backbone since both patches are the same. In this case the "A" patches bond to each other in a head-to-head manner. Conceptually, these look like the monomer/polymer shown in the upper left of the figure. The second type of monomer/polymer is shown in the lower left of Fig. 1. These monomers have two distinct bonding patches which are labeled A and "B." The bonding motif for this system is one in which the A sites bond exclusively with the B sites and bonds between the same site types (A-A and B-B) are forbidden. This motif results in chains which have a head-to-tail

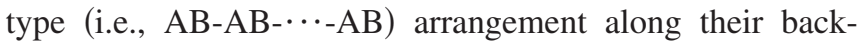
bones. The two types of bonding (head-to-head versus headto-tail) directly influence the polymer length distribution in a solution. ${ }^{55}$ This length distribution, in turn, can strongly affect the solution's physical properties. ${ }^{56}$
When a second species of monomer with a single bonding site is introduced to either of the aforementioned systems, they can associate themselves with the chain ends. Since these monomers only have one binding site, they halt the growth of the chain on the end to which they attach. Hence these units are typically referred to as "chainstoppers" or simpler, "stoppers." A conceptual view of the two types of chains with stoppers bonded to their ends is shown on the right-hand side of Fig. 1. In the first case, (upper right) the stopper may bond to either end of the chain or dimerize with another stopper and in the second, (lower right) the stoppers bond to the B-side only and are forbidden to dimerize. In these systems, the equilibrium length distribution can be well controlled by the concentration of stoppers. $^{4,57}$

\section{A. Model for associating hard-sphere fluids}

Our model for supramolecular polymers is based on a hard-sphere reference fluid in a homogeneous background medium. The pair interaction potentials between the monomers in this fluid prevent them from overlapping with each other. If we define $r_{i k}$ as the distance between the centers of two particles at locations $\mathbf{r}_{i}$ and $\mathbf{r}_{k}$, with radii $R_{i}$ and $R_{k}$, respectively, then the potential between them is $\phi_{i k}^{\mathrm{hs}}\left(r_{i k}\right)=\infty$, for $r_{i k} \leq \sigma_{i k}$ and $\phi_{i k}^{\mathrm{hs}}=0$, otherwise. In this expression we define $\sigma_{i k}=R_{i}+R_{k}$, which we shall henceforth use as a shorthand notation for the contact distance between the two particles. In addition, we define $\sigma_{i}=2 R_{i}$ as the diameter of species $i$.

At equilibrium, the bonds of a reversible polymer are constantly breaking and reforming so that a fraction of unassociated sites can be defined for the liquid. This is accounted for in the model through the theory of Wertheim, ${ }^{27-30}$ which relates the fraction of unbonded sites to the pair potential between them. In general, this potential is modeled by decorating the hard-sphere monomers on their surfaces with two attractive patches. (In the case of end stoppers, there is only one such patch per monomer.) Patches on different monomers which are designated to be complimentary to each other are given a strong, short-ranged attraction between them that represents a reversible bond. ${ }^{43,58}$ The pair potential for this associative interaction is written as 38,59

$$
\begin{aligned}
& \phi_{i k}^{j l}\left(r_{i k}, \theta_{i}^{j}, \theta_{k}^{l}\right) \\
& \quad=\left\{\begin{array}{ll}
-\epsilon_{i k}^{j l}, & r_{i k}<\sigma_{i k, c}, \\
0 & \text { otherwise, }
\end{array} \quad \theta_{i}^{j}<\theta_{i, c}^{j}, \quad \text { and } \theta_{k}^{l}<\theta_{k, c}^{l}\right.
\end{aligned}
$$

where $i$ and $k$ designate the species of the monomers and $j$ and $l$ represent the patches on their surfaces. In this equation $\sigma_{i k, c}=R_{i, c}+R_{k, c}$ is the center-to-center bonding cutoff distance, $\theta_{i, c}^{j}$ is the angle subtended by site $j$ on species $i$, and $\theta_{i}^{j}$ is the angle between a segment that passes through the center of the monomer and the center of the site and the vector $\mathbf{r}_{k}$ $-\mathbf{r}_{i}$. A schematic of the geometry for the total pair potential (hard-sphere plus association) between two bonding sites is shown in Fig. 2. The association energies for this potential $\epsilon_{i k}^{j l}$ are determined by the patch type. For the cutoff radius 


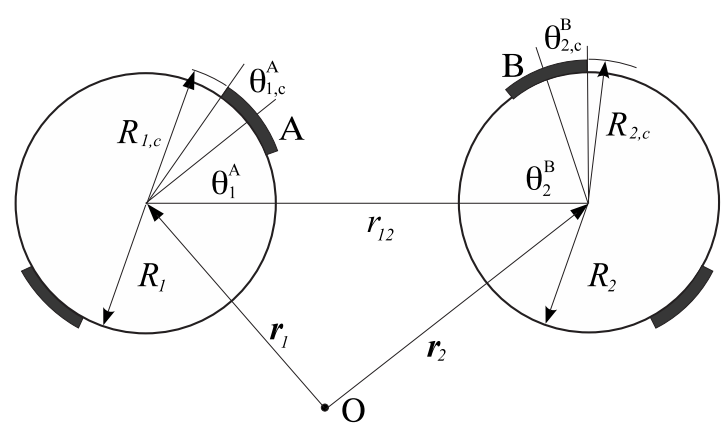

FIG. 2. Schematic of the pair potential for two monomers with the same radius, $R_{1}=R_{2}$, and single bonding patches. These two monomers bond together when they are within $2 R_{c}$ of each other and both patches $\mathrm{A}$ and $\mathrm{B}$ are within an angle $\theta_{c}$ of the axis between their centers $\mathbf{r}_{2}-\mathbf{r}_{1}$.

and angle we take $R_{i, c}=1.05 R_{i}$ and $\theta_{i, c}^{j}=\theta_{k, c}^{l}=27^{\circ}$, which are typical values for coarse-grained hydrogen bonding systems from literature. ${ }^{60}$

The computational domain for the fluid is infinite in the $x$ and $y$ directions and has parallel, planar hard walls at $z$ $=0$ and $z=L$. The potential for a species $i$ with diameter $\sigma_{i}$ interacting with these walls is

$$
\phi_{i}^{\operatorname{ext}}(z)= \begin{cases}\infty, & z<R_{i} \\ 0, & R_{i} \leq z \leq L-R_{i} \\ \infty, & z>L-R_{i},\end{cases}
$$

where $z$ is the distance from its center to the surface of a wall. For this work, where we are investigating the structure near a single wall, we have taken the distance between the walls to be large compared to a monomer unit $(L=40$ $\times \max \left\{\sigma_{i}\right\}$ ). The domain is mapped onto to a grid with small spacing $\left(\Delta z=0.05 \times \min \left\{\sigma_{i}\right\}\right)$ to maintain an accurate collocation scheme.

\section{B. Fluids density functional theory}

Density functional theory was originally developed to describe the electronic structure of atoms using a variational calculus. $^{61}$ It was first employed for classical fluids in the late 1970 s to describe liquid structure and behavior at interfaces. ${ }^{62}$ The theorem underlying FDFT states that the equilibrium properties of a fluid system can be calculated from a functional $\Omega\left[\left\{\rho_{i}\right\}\right]$ of the set of inhomogeneous density distributions $\left\{\rho_{i}(\mathbf{r})\right\}$ of its constituent species. Given a set of pair interactions and external potentials, this functional has a minimum value when $\rho_{i}(\mathbf{r})=\widetilde{\rho}_{i}(\mathbf{r})$ for each of the species $i$, where $\tilde{\rho}_{i}(\mathbf{r})$ are the equilibrium density profiles of each species. The functional $\Omega\left[\widetilde{\rho}_{i}\right]$ of the equilibrium density profiles corresponds to the grand potential of an open $(\mu V T)$ system. The grand potential for the fluid system in this work is expressed mathematically as

$$
\begin{aligned}
\Omega\left[\left\{\rho_{i}(\mathbf{r})\right\}\right]= & F^{\mathrm{id}}\left[\left\{\rho_{i}(\mathbf{r})\right\}\right]+F^{\mathrm{hs}}\left[\left\{\rho_{i}(\mathbf{r})\right\}\right]+F^{\mathrm{as}}\left[\left\{\rho_{i}(\mathbf{r})\right\}\right] \\
& +\sum_{i} \int d \mathbf{r}^{\prime} \rho_{i}\left(\mathbf{r}^{\prime}\right)\left[\phi_{i}^{\mathrm{ext}}\left(\mathbf{r}^{\prime}\right)-\mu_{i}\right]
\end{aligned}
$$

where $F^{\text {id }}$ is the ideal Helmholtz free energy, and $F^{\text {hs }}$ and $F^{\text {as }}$ are the excess free energies arising from the hard-sphere and associative interactions between the particles, respectively.
The sum of these three terms provides a coarse-grained description of the properties of the fluid as a functional of its constituent densities. The summation term in Eq. (3) is the Legendre transformation in which $\mu_{i}$ are the species' chemical potentials and $\phi_{i}^{\text {ext }}(\mathbf{r})$ are external fields acting on each species due to the presence of the hard walls in the system. Like the free energy, the chemical potential of each species can also be divided into its ideal and excess parts (due to hard-sphere and association interactions) and written as $\mu_{i}$ $=\mu_{i}^{\mathrm{id}}+\mu_{i}^{\mathrm{hs}}+\mu_{i}^{\mathrm{as}}$, where the contribution type is denoted by the superscripts.

Minimization of Eq. (3) with respect to the density profiles of each fluid component produces a set of EulerLagrange (EL) equations which must be solved for all points on the grid in the domain. The EL equations are calculated at constant chemical potential $\mu$, volume $V$, and temperature $T$, i.e., $\left(\delta \Omega\left[\widetilde{\rho}_{i}\right] / \delta \widetilde{\rho}_{i}\right)_{\mu, V, T}=0$. These nonlinear, integrodifferential equations are solved with a bound-constrained quasi-Newton method. $^{63,64}$

Our choice to use FDFT was motivated by the successes of the various SAFT theories for polymer systems. ${ }^{65}$ Since our theory reduces to a SAFT theory in the bulk, it provides us with a boundary condition to work from. In addition, we expect the method to perform well for the higherconcentration regimes because there the chains, which are constantly forming and breaking under equilibrium conditions, exhibit near-ideal conformation statistics. These ideal statistics imply that a certain cancellation of correlation hole effects exists, as is discussed in many textbooks on polymer physics. ${ }^{15}$ At lower concentrations, i.e., low and so-called semidilute concentration regimes, this cancellation fails. To account for this a more sophisticated theory would be needed. Such a theory would need to treat the various sites on each monomer as dependent, and would require an intramolecular correlation function. Investigation of the form of this function could lead to an interesting extension of this work, but is not discussed here.

\section{Hard-sphere fluid}

The Helmholtz free energy functionals for a fluid are independent of the external potential and are, in general, unknown. The only functional which is known exactly is that of the ideal gas, given by

$$
F^{\mathrm{id}}\left[\left\{\rho_{i}(\mathbf{r})\right\}\right]=k_{B} T \sum_{i} \int d \mathbf{r}^{\prime} \rho_{i}\left(\mathbf{r}^{\prime}\right)\left[\ln \left(\Lambda_{i}^{3} \rho_{i}\left(\mathbf{r}^{\prime}\right)\right)-1\right],
$$

where $k_{B}$ is the Boltzmann constant, $T$ is the temperature, and $\Lambda_{i}$ is the thermal wavelength of the component $i$.

To compute the hard-sphere excess free energy, we use a fundamental measure theory ${ }^{66}$ (FMT) of Rosenfeld. FMT models are based on the geometric interactions between the fluid components. The excess free energy functional for such a system is given by

$$
F^{\mathrm{hs}}\left[\left\{\rho_{i}(\mathbf{r})\right\}\right]=k_{B} T \int d \mathbf{r}^{\prime} \Phi^{\mathrm{hs}}\left[\left\{n_{\alpha}\left(\mathbf{r}^{\prime}\right)\right\}\right],
$$

where the energy density for the hard-sphere system, $\Phi^{\text {hs }}$, is a functional of a set of inhomogeneous weighted densities of 
the system, $n_{\alpha}$. These weighted densities are based on the deconvolution of the two-body Mayer- $f$ function $^{67}$ for hardspheres, into a sum of functions of the radii of the individual particle types. ${ }^{66}$ We write the densities as

$$
n_{\alpha}(\mathbf{r})=\sum_{i} \int d \mathbf{r}^{\prime} \rho_{i}\left(\mathbf{r}^{\prime}\right) \omega_{i}^{(\alpha)}\left(\mathbf{r}-\mathbf{r}^{\prime}\right)
$$

where $\omega_{i}^{(\alpha)}$ are the four scalar and two vector weighting functions, $\alpha \in\{0,1,2,3, V 1, V 2\}$. Essentially, these weighting functions are based on the fundamental geometric properties of the species, i.e., radius $R_{i}=\sigma_{i} / 2$, surface area, and volume. They are

$$
\begin{aligned}
& \omega_{i}^{(2)}(\mathbf{r})=\delta\left(R_{i}-|\mathbf{r}|\right), \quad \omega_{i}^{(3)}(\mathbf{r})=\theta\left(R_{i}-|\mathbf{r}|\right), \\
& \omega_{i}^{(0)}(\mathbf{r})=\frac{\omega_{i}^{(2)}(\mathbf{r})}{4 \pi R_{i}^{2}}, \quad \omega_{i}^{(1)}(\mathbf{r})=\frac{\omega_{i}^{(2)}(\mathbf{r})}{4 \pi R_{i}} \\
& \omega_{i}^{(V 2)}(\mathbf{r})=\frac{\mathbf{r}}{r} \delta\left(R_{i}-|\mathbf{r}|\right), \quad \omega_{i}^{(V 1)}(\mathbf{r})=\frac{\omega_{i}^{(V 2)}(\mathbf{r})}{4 \pi R_{i}},
\end{aligned}
$$

where $\delta(\mathbf{r})$ and $\theta(\mathbf{r})$ denote the Dirac delta function and Heaviside step function, respectively.

Several forms of the hard-sphere energy density, $\Phi^{\mathrm{hs}}$, can be found in literature, each one based on a different Ansatz. ${ }^{66,68-71}$ The specific functional used in this work is due to Rosenfeld ${ }^{66}$ and is given by

$$
\begin{aligned}
\Phi^{\mathrm{hs}}= & -n_{0} \ln \left(1-n_{3}\right)+\frac{n_{1} n_{2}-n_{V 1} \cdot n_{V 2}}{1-n_{3}} \\
& +\frac{n_{2}^{3}-3 n_{2} n_{V 2} \cdot n_{V 2}}{24 \pi\left(1-n_{3}\right)^{2}} .
\end{aligned}
$$

Far from any interfaces, this energy density corresponds to that of a Percus-Yevick type hard-sphere fluid. In onedimension this equation exactly corresponds to a hard-rod fluid. ${ }^{69}$ This can be seen through the equation of state for the pressure of the fluid in the bulk where $n_{V 1}=n_{V 2}=0$. It is

$$
\beta p=\frac{n_{0}}{1-n_{3}}+\frac{n_{1} n_{2}}{\left(1-n_{3}\right)^{2}}+\frac{n_{2}^{3}}{12 \pi\left(1-n_{3}\right)^{3}},
$$

which corresponds to the Percus-Yevick equation of state with $\beta=1 / k_{B} T$.

\section{Association perturbation}

We treat the monomer-monomer attractions with an inhomogeneous first-order thermodynamic perturbation theory. $^{27-30,43}$ The idea behind this theory is to divide the fluid density into its bonded and unbonded constituents, subtract off a reference part, and rearrange the terms so that the excess free energy correction to the reference fluid can be expressed in terms of its fraction of unbonded sites. The excess free energy for association of a fluid with $n$ types of monomers and $s$ types of bonding sites can be expressed as $^{38,58}$

$$
\begin{aligned}
F^{\mathrm{as}}\left[\left\{\rho_{i}(\mathbf{r})\right\}\right]= & k_{B} T \int d \mathbf{r}^{\prime} \sum_{i=1}^{n} \rho_{i}\left(\mathbf{r}^{\prime}\right) \\
& \times \sum_{j=1}^{s} S_{i}^{j}\left(\ln X_{i}^{j}\left(\mathbf{r}^{\prime}\right)-X_{i}^{j}\left(\mathbf{r}^{\prime}\right) / 2+1 / 2\right),
\end{aligned}
$$

where $S_{i}^{j}$ is the number of sites of type $j$ on a monomer of species $i$ and $X_{i}^{j}(\mathbf{r})$ is the fraction of unassociated bonding sites on monomers at spatial location $\mathbf{r}^{60}$ This fraction is given by

$$
\begin{aligned}
X_{i}^{j}(\mathbf{r})= & {\left[1+\int d \mathbf{r}^{\prime} \sum_{k=1}^{n} \rho_{k}\left(\mathbf{r}^{\prime}\right)\right.} \\
& \left.\times \sum_{l=1}^{s} S_{k}^{l} X_{k}^{l}\left(\mathbf{r}^{\prime}\right) \Delta_{k i}^{l j}\left(\mathbf{r}, \mathbf{r}^{\prime} ; \sigma_{i}, \sigma_{k}\right)\right]^{-1} .
\end{aligned}
$$

The factor $\Delta_{k i}^{l j}\left(\mathbf{r}, \mathbf{r}^{\prime} ; \sigma_{i}, \sigma_{k}\right)$ for a hard-sphere reference fluid with a narrow square-well association site is ${ }^{60}$

$$
\Delta_{k i}^{l j}\left(\mathbf{r}, \mathbf{r}^{\prime} ; \sigma_{i}, \sigma_{k}\right)=4 \pi \hat{g}_{k i}^{\mathrm{hs}}\left(\mathbf{r}, \mathbf{r}^{\prime}\right) A_{k i}^{l j}\left[\exp \left(\frac{\epsilon_{k i}^{l j}}{k_{B} T}\right)-1\right],
$$

where $A_{k i}^{l j}$ is a geometric factor which represents an entropic penalty for associations, $\hat{g}_{k i}^{\mathrm{hs}}\left(\mathbf{r}, \mathbf{r}^{\prime}\right)$ is the contact value of the two point hard-sphere correlation function, and the bracketed portion is the Mayer-F function for the angular part of the pair potential given in Eq. (1). The geometric factor is calculated using

$$
A_{k i}^{l j}=\frac{\left(1-\cos \theta_{i, c}^{j}\right)\left(1-\cos \theta_{k, c}^{l}\right)}{4} \sigma_{k i}^{2}\left(\sigma_{i k, c}-\sigma_{k i}\right),
$$

where the cutoff diameter and angles $\left(\sigma_{i k, c}\right.$ and $\left.\theta_{k, c}\right)$ are defined in Sec. II. This term is analogous to the average amount of volume available for bonding between a given pair of patches. $^{49}$

The two point hard-sphere correlation functions, which are unknown in general, ${ }^{35}$ were approximated using the geometric mean of the single point correlation functions, ${ }^{38}$ i.e., $\hat{g}_{k i}^{\mathrm{hs}}\left(\mathbf{r}, \mathbf{r}^{\prime}\right)=\left[\hat{g}_{k i}^{\mathrm{hs}}(\mathbf{r}) \times \hat{g}_{k i}^{\mathrm{hs}}\left(\mathbf{r}^{\prime}\right)\right]^{1 / 2}$. The single point pair correlation functions, $\hat{g}_{k i}(\mathbf{r})$, used in this geometric mean have positional arguments due to the spatially varying densities in the computational domain. They can be calculated either using locally averaged densities and the multispecies PercusYevick equation, ${ }^{72,73}$ or directly from the FMT weighted densities. $^{45}$ In the latter case we have ${ }^{74}$

$$
\begin{aligned}
\hat{g}_{k i}^{\mathrm{hs}}(\mathbf{r})= & \frac{1}{1-n_{3}(\mathbf{r})}+\left(\frac{\sigma_{i} \sigma_{k}}{\sigma_{i}+\sigma_{k}}\right) \frac{n_{2}(\mathbf{r}) \zeta(\mathbf{r})}{2\left(1-n_{3}(\mathbf{r})\right)^{2}} \\
& +\left(\frac{\sigma_{i} \sigma_{k}}{\sigma_{i}+\sigma_{k}}\right)^{2} \frac{n_{2}(\mathbf{r})^{2} \zeta(\mathbf{r})}{12\left(1-n_{3}(\mathbf{r})\right)^{3}},
\end{aligned}
$$

where $\zeta(\mathbf{r})=1-n_{V 2} \cdot n_{V 2} / n_{2}^{2}$. At the densities considered herein, the difference between the two methods was found to be negligible; however, the latter method was found to give more accurate values for the pressure at higher densities. Also it is more computationally efficient overall because the density averaging required to compute the former method requires additional integrals over the grid. ${ }^{75}$ 


\section{E. Chemical potentials}

Since the system is in equilibrium, the chemical potential can be computed for each species from the derivatives of the free energy terms (ideal or excess) with respect to the bulk density of that species, i.e.,

$$
\mu_{i}^{\mathrm{X}}=k_{B} T\left[\frac{\partial\left(F^{\mathrm{X}, \mathrm{bulk} / V)}\right.}{\partial \rho_{i}^{\text {bulk }}}\right]_{\forall \rho_{j} \neq \rho_{i}, V, T}
$$

For the ideal gas term of a species $i$ we find

$$
\mu_{i}^{\text {id }}=k_{B} T \ln \left(\rho_{i}^{\text {bulk }} \Lambda_{i}^{3}\right)
$$

The excess hard-sphere contribution to the chemical potential for each species is calculated using

$$
\mu_{i}^{\mathrm{hs}}=k_{B} T \sum_{\alpha} \frac{\partial \Phi^{\mathrm{hs}, \mathrm{bulk}}}{\partial n_{\alpha}} \frac{\partial n_{\alpha}}{\partial \rho_{i}^{\text {bulk }}},
$$

where the sum of the nonlocal density derivatives have replaced the species density derivatives through the chain rule. The excess chemical potential due to association can be computed from the derivative of the bulk free energy with respect to the density, ${ }^{43}$ i.e.,

$$
\begin{aligned}
\beta \mu_{i}^{\mathrm{as}}= & \sum_{j=1}^{s} S_{i}^{j}\left[\ln X_{i}^{(j, \text { bulk })}-\frac{X_{i}^{(j, \text { bulk })}}{2}+\frac{1}{2}\right] \\
& +\sum_{k=1}^{n} \sum_{l=1}^{s} S_{k}^{l} \rho_{k}^{\text {bulk }}\left(\frac{1}{X_{k}^{(l, \text { bulk })}}-\frac{1}{2}\right) \frac{\partial X_{k}^{(l, \text { bulk })}}{\partial \rho_{i}^{\text {bulk }}} .
\end{aligned}
$$

This form of the function is rather cumbersome since it requires the solution of Eq. (11) and its derivatives. ${ }^{59}$ However, it is possible to rearrange the excess Helmholtz free energy for association into an equivalent quadratic form. ${ }^{76}$ This form simplifies the expression for the chemical potential to one only requiring the derivatives of the function $\Delta_{i k}^{j l}$ with respect to density. With this technique, the chemical potential term due to association is

$$
\begin{aligned}
\beta \mu_{m}^{\text {as }}= & \sum_{j=1}^{s} S_{m}^{j} \ln X_{m}^{(j, \text { bulk })}-\frac{1}{2} \sum_{i=1}^{n} \sum_{k=1}^{n} \sum_{j=1}^{s} \sum_{l=1, l \neq j}^{s} \rho_{i}^{\text {bulk }} \\
& \times \rho_{k}^{\text {bulk }} S_{i}^{j} S_{k}^{l} X_{i}^{(j, \text { bulk })} X_{k}^{(l, \text { bulk })} \frac{\partial \Delta_{i k}^{j l \text { bulk }}}{\partial \rho_{m}^{\text {bulk }}} .
\end{aligned}
$$

\section{F. Solution of the equations}

Substituting Eqs. (4), (5), and (10) into Eq. (3) and performing the functional derivative with respect to the fluid component densities, we arrive at the Euler-Lagrange equations for the system. This system contains $m$ equations-one for each species of monomer present. These equations are

$$
\begin{aligned}
\ln \left(\rho_{m}(\mathbf{r}) \Lambda_{m}^{3}\right) & \\
= & \left(\mu_{m}-\phi_{m}^{\mathrm{ext}}(r)\right) / k_{B} T-\int d \mathbf{r}^{\prime} \sum_{\alpha} \frac{\partial \Phi^{\mathrm{hs}}\left[\left\{n_{\alpha}\left(\mathbf{r}^{\prime}\right)\right\}\right]}{\partial n_{\alpha}} \\
& \times \omega^{(\alpha)}\left(\mathbf{r}-\mathbf{r}^{\prime}\right)-\sum_{j=1}^{s} S_{m}^{j} \ln X_{m}^{j}(\mathbf{r}) \\
& +\frac{1}{2} \sum_{i=1}^{n} \sum_{k=1}^{n} \sum_{j=1}^{s} \sum_{l=1, l \neq j}^{s} S_{i}^{j} S_{k}^{l} \int d \mathbf{r}^{\prime} \int d \mathbf{r}^{\prime \prime} \rho_{i}\left(\mathbf{r}^{\prime}\right) \rho_{k}\left(\mathbf{r}^{\prime \prime}\right) \\
& \times X_{i}^{j}\left(\mathbf{r}^{\prime}\right) X_{k}^{l}\left(\mathbf{r}^{\prime \prime}\right) \frac{\partial \Delta_{i k}^{j l}\left(\mathbf{r}^{\prime}, \mathbf{r}^{\prime \prime}\right)}{\partial \rho_{m}(\mathbf{r})} .
\end{aligned}
$$

To solve these equations we must first choose bulk densities $\rho_{m}^{\text {bulk }}$ and bonding site parameters $R_{i, c}, \theta_{i, c}^{j}$, and $\epsilon_{i k}^{j l}$ and calculate the chemical potential terms for these parameters (scaled such that $\Lambda_{m}=1$ and $k_{B} T=1$ ).

An important check for self-consistency of the hardsphere functional is the contact sum rule. ${ }^{67}$ This rule requires that for an unconfined hard-sphere fluid, the sum of the number densities for all the species at the point where the fluid contacts a hard wall should be equal to the bulk pressure of the system. We write this as

$$
\beta p=\sum_{i} \rho_{i}\left(z=R_{i}\right) .
$$

At low to mid-densities $(0 \leq \rho \leq 0.4)$, such as the ones we are trying to model in this work Eq. (8) performs well, with errors in the $0 \%-10 \%$ range. In order to go to higher densities, we would need to implement a version of the hardsphere functional which is based on a better behaved equation of state such as the Mansoori-Carnahan-StarlingLeland equation. ${ }^{77}$ The White Bear functional would be an ideal candidate. ${ }^{70}$

\section{RESULTS AND ANALYSIS}

In this section, we show results from the application of the theory described above to several systems of supramolecular polymers near a hard wall. First, we present two monomeric fluids, each with two bonding sites, but with different bonding affinities. One fluid (denoted by AA) is constituted from monomers with two indistinguishable bonding patches labeled A. These sites are attractive to A sites on other monomers and give rise to chains with no backbone monomer orientation. The other fluid (denoted by $\mathrm{AB}$ ) is made up of monomers containing two bonding groups labeled $\mathrm{A}$ and $\mathrm{B}$ and the formation of $\mathrm{AB}$ bonds is allowed while AA and BB bonds are forbidden. This schema produces chains with monomers oriented along their backbones. These two model systems serve as a calibration and validation of the FDFT against previous results for such fluids. ${ }^{22,60}$

In addition to monomeric systems, we examine the effects of adding a second species of chain-stoppers. These stoppers have a bonding site labeled $\mathrm{A}$, which is given the same bonding criteria as the A patch on the two-site species it is mixed with. They have the effect of causing chains to terminate at one end and allow for growth on the A sides of 


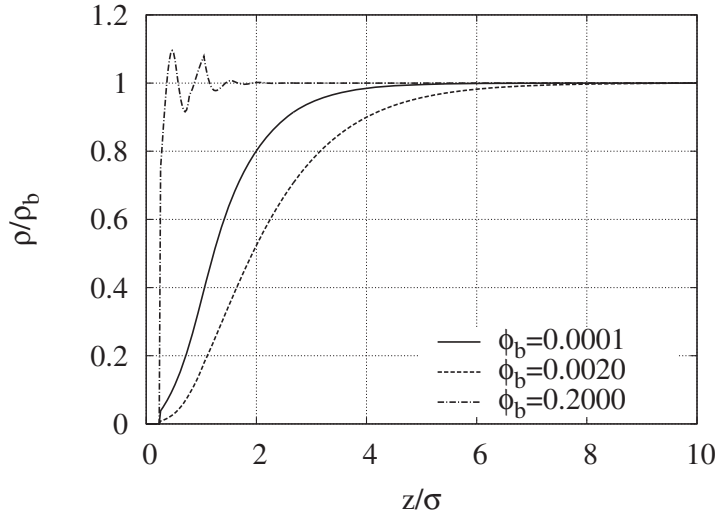

FIG. 3. Normalized density profiles for a monomeric associating fluid with two sites labeled A and B. Only AB bonds are allowed with strength of $\epsilon$ $=20 k_{B} T$. The solid, dashed, and dotted lines correspond to packing fractions $\phi_{b}=0.0001,0.0020$, and 0.2000 , respectively. Note that the adsorption profiles are nonmonotonic in monomer concentration (see text.)

the chains. By changing the concentration of stoppers it is possible to control the average equilibrium chain length in the solution.

\section{A. Monomeric AA and $A B$ solutions}

For monomeric systems we calculated the density profiles for packing fractions ranging from $\phi_{b}=10^{-5}$ to $\phi_{b}=0.2$, where $\phi_{b}=\pi \rho^{\text {bulk }} \sigma^{3} / 6$. Three such profiles from an AB system are plotted in Fig. 3. In these calculations the bonding strengths were $\epsilon_{11}^{A A}=\epsilon_{11}^{B B}=0$ and $\epsilon_{11}^{A B}=\epsilon_{11}^{B A}=\epsilon=20 k_{B} T$ (with $\left.k_{B} T=1\right)$. The bonding site potential [Eq. (1)] was defined with $R_{1 c}=0.525$ and $\theta_{1, c}=27^{\circ}$. As can be seen in the figure, the density profiles follow a nonmonotonic behavior with respect to increasing concentration.

At the lowest concentration plotted in Fig. 3, $\left(\phi_{b}\right.$ $=0.0001$ solid line) the monomers are spaced far apart which leads to short chains. At $\phi_{b}=0.002$ (dashed line) the chains become longer and their entropy makes the depletion effect larger. At the highest concentration $\left(\phi_{b}=0.2\right.$ dotted line $)$ packing effects become dominant and the fluid becomes ordered at the wall. In this case, the second peak is larger than that of a hard-sphere fluid due to the bonding that occurs between the first and second layers of the fluid.

The difference between $\mathrm{AB}$ and $\mathrm{AA}$ systems is plotted in Fig. 4. For both systems $\epsilon=20 k_{B} T$ but the AA system provides more chances for bonding sites to connect at a given concentration. This extra bonding magnifies the effect of depletion over the $\mathrm{AB}$ system. This extra depletion is caused by the AA system having a longer average chain size as we shall demonstrate.

The bulk average chain lengths of monomeric AA and $\mathrm{AB}$ systems with different associating strengths are plotted as a function of monomer volume fraction in Fig. 5. As can be seen in this figure, for a given concentration and association strength, the chains in the AA systems are longer than those in the AB systems. Also at $\epsilon=20 k_{B} T$ the scaling exponent of length versus concentration approaches $1 / 2$, which is typical of a monodisperse covalently bonded polymer in a random coil configuration. We note that at higher packing fractions $\left(\phi_{b} \geq 0.8\right)$ the curve begins to deviate from the

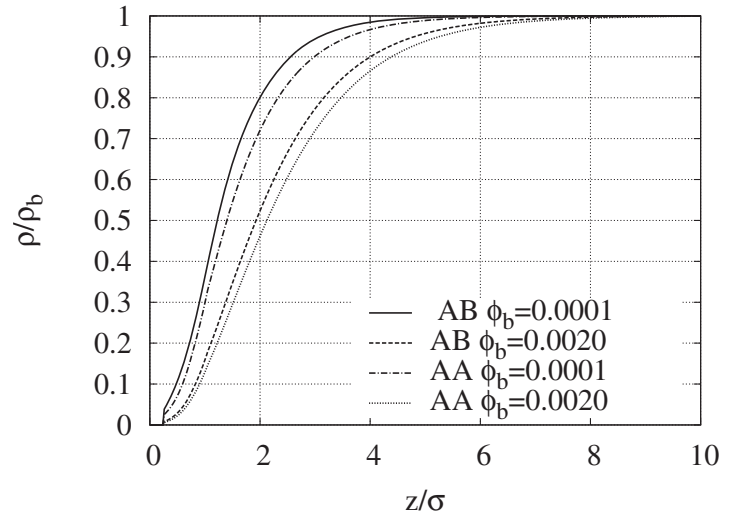

FIG. 4. Comparison of the normalized density profiles of two-site monomer systems with AA bonds and $\mathrm{AB}$ bonds in the dilute $\left(\phi_{b}=0.0001\right)$ and marginal regimes $\left(\phi_{b}=0.002\right)$. In both cases the bonding strength is $\epsilon=20 k_{B} T$. The AA systems (short dashed and dotted lines) show more depletion from the surface than the $\mathrm{AB}$ systems (solid and long dashed lines) at the same fluid density.

power law due to packing effects. Since the particles begin to have more contacts $[\hat{g}(\mathbf{r})$ increases] they tend to adopt the more energetically favorable bonded configurations and hence the chain lengths scale with a slightly larger exponent.

The average chain length for a particular system can be expressed by recognizing that the number of unbonded sites $X$ is related to the Flory parameter $p$ by $X=1-p{ }^{78,79}$ Given this relationship, the average chain length can be computed directly. For identical heterocomplimentary bonding sites (AB), $\Delta=\Delta_{11}^{12}=\Delta_{11}^{21}$ and $\Delta_{11}^{11}=\Delta_{11}^{22}=0$ and $S_{1}^{1}=S_{1}^{2}=1$, and $X$ $=X_{1}^{1}=X_{1}^{2}$. Defining $\rho=\rho_{1}^{\text {bulk }}$, the fraction of unbonded sites for the system in the bulk region of the computational domain is given by $\langle N\rangle_{w}=\sqrt{1+4 \rho \Delta} \approx 2 \sqrt{\rho \Delta}$ for $\rho \Delta \gg 1$. Similarly, for self-complimentary systems, $\langle N\rangle_{w}=\sqrt{1+8 \rho \Delta}$. Comparing these results to a chemical theory for bonding, ${ }^{57}$ we find that it is possible to directly estimate the equilibrium chain length from the Mayer-F function and the physical properties of the monomers themselves. In short, $\Delta$ is directly mappable to the association constant.

As was stated earlier, the monomeric systems exhibit a nonmonotonic depletion layer thickness with respect to

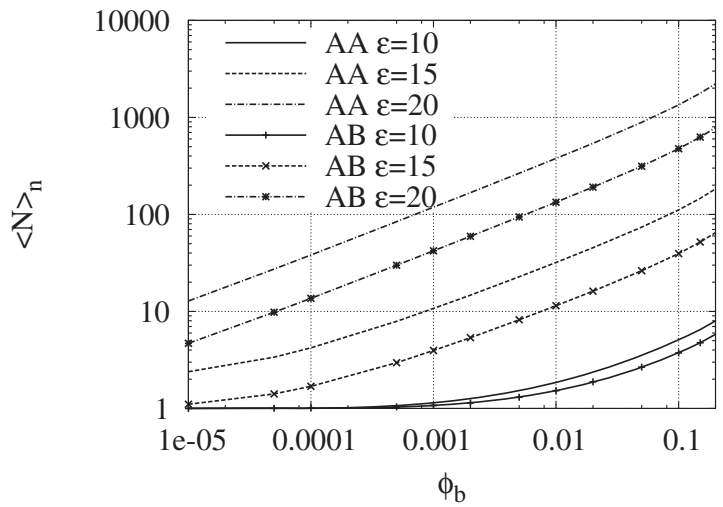

FIG. 5. Average chain lengths for various monomeric AA and AB systems. Three different association strengths are shown $\epsilon / k_{B} T=\{10,15,20\}$. In all cases the AA system has longer chains than the AB system with the same parameters. At $\epsilon=20 k_{B} T$ the chains begin to have the same scaling exponent as regular covalently bonded polymers, i.e., $\langle N\rangle_{n} \sim \phi_{b}^{1 / 2}$. The scaling increases beyond $\phi \approx 0.8$ because of packing effects. 


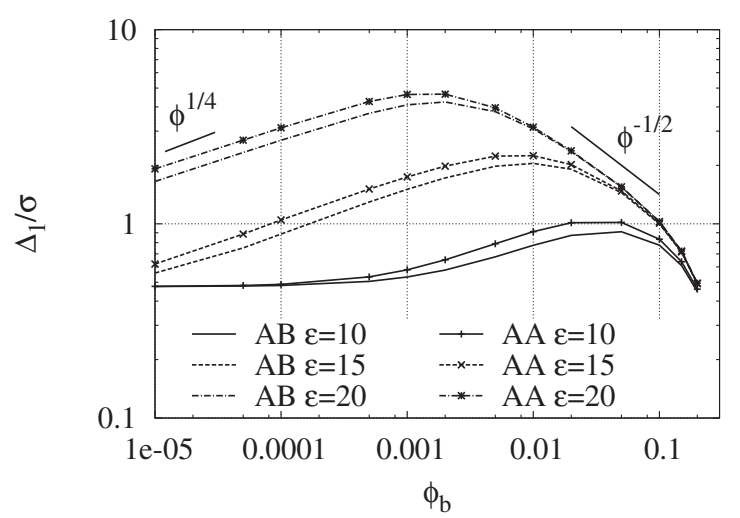

FIG. 6. Depletion layer thickness as a function of bulk concentration for AA and $\mathrm{AB}$ solutions for various association energies. Note for $\epsilon=10 k_{B} T$ the system stops forming bonds at low concentrations and approaches an asymptotic value of $\sigma / 2$.

monomer density. To understand why this is so, we can calculate the excess adsorption of the system and use it to infer the depletion length. The surface excess adsorption per unit area for a fluid component $i$ near the wall is defined as ${ }^{80}$

$$
\Gamma_{i}=\sigma_{i}^{3} \int_{0}^{L / 2} d z\left[\rho_{i}(z)-\rho_{i}^{\text {bulk }}\right]
$$

where the integral is taken from the wall to the center of the box. The depletion length for an unconfined fluid near a single surface can be defined as ${ }^{22}$

$$
\Delta_{1}=-\frac{\Gamma}{\rho_{b}}
$$

where $\rho_{b}$ is the bulk density. This quantity is plotted for both $\mathrm{AA}$ and $\mathrm{AB}$ monomeric systems in Fig. 6. In the dilute regime the depletion length should be determined by the average radius of gyration of the polymers. ${ }^{16}$ In this case the chains have random walk statistics, i.e.,

$$
\Delta_{1} \sim\langle N\rangle^{1 / 2} \sim \phi_{b}^{1 / 4} \exp \left(\frac{\epsilon}{4 k_{B} T}\right)
$$

This scaling can be observed in Fig. 6. In the marginal regime, the polymer depletion length is set by the bulk correlation length. This is given by

$$
\Delta_{1} \sim\langle\xi\rangle \sim \phi_{b}^{-1 / 2}
$$

and is plotted in Fig. 6. Finally, the scaling in the concentrated regime follows $\Delta_{1} \sim \phi_{b}^{-1}$, (also plotted in Fig. 6) as it should for a densely packed system. As can be seen in this figure, the system follows the well known scaling rules as a function of density. ${ }^{16}$ There is a maximum which corresponds to the crossover concentration between the dilute and marginal regions. These results are in good agreement with those of van der Gucht ${ }^{22}$ for the scaling behavior, but the overall depletion lengths in this work are generally lower in magnitude. This is likely due to the different representations of the fluid (e.g., lattice versus off-lattice).

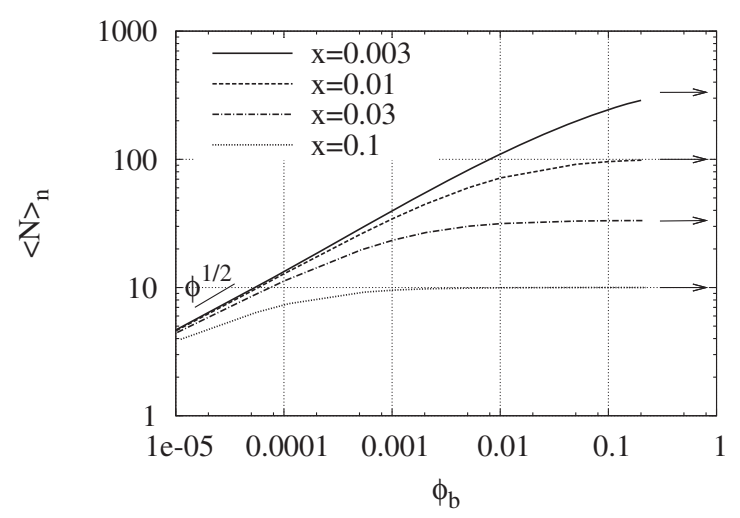

FIG. 7. Average chain length in the bulk as a function of volume fraction for binary systems with chain-stoppers. The arrows on the right indicate the asymptotic average chain length $\sim 1 / x$, where $x$ is the fraction of stoppers present in the system. The line above the data at the left of the figure shows $\phi_{b}^{1 / 2}$.

\section{B. Binary solutions of $A B$ monomers and $B$ chain-stoppers}

The behavior of $\mathrm{AB}$ systems can be further controlled through the addition of monofunctional monomers with a single B site. These monomers bond to the A site on the bifunctional monomers and create nonfunctional chain ends. Hence, they are called chain-stoppers. These chain-stoppers change the system behavior markedly by limiting the amount of polymerization. In short, the average length of chains in the system decreases as a function of increasing stopper fraction. This decrease in chain length means that the number of chains in the system increases.

The effects of stoppers can be analyzed with a technique similar to that used in condensation polymerization. ${ }^{78}$ The stopper fraction is defined as $x=\rho_{2} /\left(\rho_{1}+\rho_{2}\right)$, where $\rho_{1}$ and $\rho_{2}$ are the densities of $\mathrm{AB}$ monomers and $\mathrm{B}$ monomers, respectively. With this definition, the average chain length of the system can be written as ${ }^{4}$

$$
\langle N(x)\rangle_{n} \simeq \frac{\langle N(0)\rangle_{n}}{1-x\left(1+\langle N(0)\rangle_{n}\right)},
$$

where $\langle N(0)\rangle_{n}$ is the average chain length in a system without stoppers. When $\langle N(0)\rangle_{n}$ is large this value approaches $1 / x$. This suggests that the average chain length can be made independent of the monomer density for a system.

The average chain length for binary systems containing two-site $\mathrm{AB}$ and single site $\mathrm{B}$ monomers is plotted as a function of total volume fraction in Fig. 7. In this figure four different stopper concentrations $x=\{0.003,0.01,0.03,0.1\}$ are shown. At low concentrations the chains grow with concentration at the usual rate $(N$ $\sim \phi_{b}^{1 / 2}$ ) but as the concentration increases, the average chain length becomes independent of it.

The effect of the decreased average chain length in a binary system can be observed in the depletion length. Figure 8 contains a plot of depletion length versus total bulk volume fraction of monomers. The upper envelope curve in this plot is that of a pure AB system (i.e., $x=0$ ). The addition of chain-stoppers causes the depletion length to decrease. It also appears to decrease the overlap concentration of the sys- 


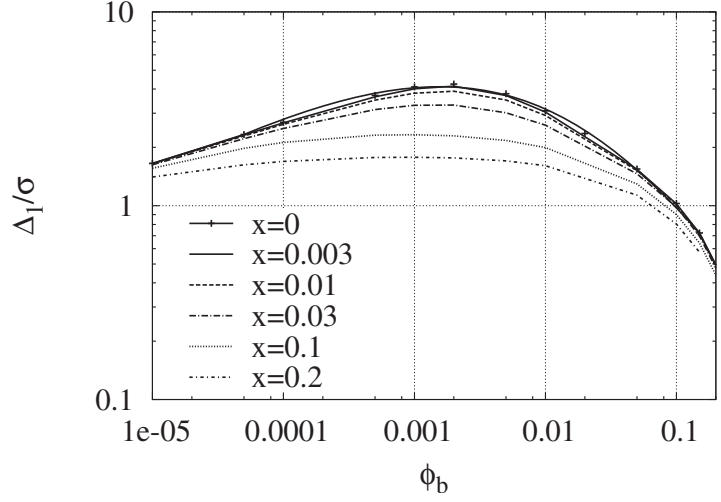

FIG. 8. Depletion layer thickness as a function of volume fraction for binary systems with chain-stoppers. The envelope curve at the top is that of a two bonding site system without end stoppers.

tem as the maximum shifts to the left. However, for this type of system, the maximum does not correspond with the overlap concentration.

For a system with very strong association, the curve should grow as $\Delta_{1} \sim \phi_{b}^{1 / 4}$ until the chain length saturates to the fraction of stoppers. At this point it remains flat until the overlap concentration is reached, at which point it would turn to $\Delta_{1} \sim \phi_{b}^{-1 / 2}$. This effect can be seen in the lowest curve of Fig. 8 which has a stopper fraction of $x=0.2$.

\section{CONCLUSION}

We have shown that FDFT can be used to calculate the properties of reversible supramolecular polymers. Unfortunately, to the best of our knowledge, no simulation or experimental results on the density profiles of nonadsorbing living polymers near a surface are available in the literature. The most relevant experimental results available are, perhaps, our work on depletion interaction between surfaces immersed in solutions of supramolecular polymers. ${ }^{4,81}$ Direct comparison between these results and our calculations would be, at best, speculative since the experiment does not provide density profiles.

On the other hand, it is possible to compare our system to previous lattice- based mean-field calculations. For monomeric systems with two bonding sites we have established a correspondence between those calculations and the new FDFT approach. Particularly, the new technique was shown to have correct scaling behavior from low concentrations all the way up to concentrations where packing effects begin to take place. At higher densities, we observe ordering due to packing of the monomers as well as a stronger than $\phi^{1 / 2}$ dependency of the average degree of polymerization.

The theory was then extended to study binary systems containing monomers with two bonding sites and chainstoppers which have only a single bonding site. These chainstoppers can be used to control the chain length, which in turn, set the depletion length for the system. We hope to extend this work to study these fluid systems confined between two surfaces in order to mimic the gap between interacting colloidal particles. Further, we would like to investigate the effects of placing binding groups on the surfaces which act as chain ends for supramolecular polymers. This should lead to a better understanding of the relative effects of entropy and binding energy for functionalized colloidal systems.

\section{ACKNOWLEDGMENTS}

Funding for this work was provided by the T. U. Delft visiting scholar grant. E.S.M. would like to thank Professor Stephen Picken from T. U. Delft and Professor Paul van der Schoot from T. U. Eindhoven for thier insightful discussions about this work.

${ }^{1}$ L. Brunsveld, B. J. B. Folmer, E. W. Meijer, and R. P. Sijbesma, Chem. Rev. (Washington, D.C.) 101, 4071 (2001).

${ }^{2}$ A. W. Bosnian, L. Brunsveld, B. J. B. Folmer, R. P. Sijbesma, and E. W. Meijer, Macromol. Symp. 201, 143 (2003).

${ }^{3}$ R. P. Sijbesma, F. H. Beijer, L. Brunsveld, B. J. B. Folmer, J. H. K. Hirschberg, R. F. M. Lange, J. K. L. Lowe, and E. W. Meijer, Science 278, 1601 (1997).

${ }^{4}$ W. Knoben, N. A. M. Besseling, and M. A. Cohen Stuart, Phys. Rev. Lett. 97, 068301 (2006).

${ }^{5}$ R. L. Scott, J. Phys. Chem. 69, 261 (1965).

${ }^{6}$ J. C. Wheeler, S. J. Kennedy, and P. Pfeuty, Phys. Rev. Lett. 45, 1748 (1980).

${ }^{7}$ S. J. Kennedy and J. C. Wheeler, J. Phys. Chem. 88, 6595 (1984).

${ }^{8}$ M. E. Cates and S. J. Candau, J. Phys.: Condens. Matter 2, 6869 (1990).

${ }^{9}$ A. Ekani-Nkodo, A. Kumar, and D. Fygenson, Phys. Rev. Lett. 93, 268301 (2004).

${ }^{10}$ J. Xu, T. H. LaBean, and S. L. Craig, in Supramolecular Polymers, edited by A. Ciferri (CRC, Boca Raton, FL, 2004).

${ }^{11}$ P. T. Cummings and G. Stell, Mol. Phys. 51, 253 (1984).

${ }^{12}$ P. T. Cummings and G. Stell, Mol. Phys. 55, 33 (1985).

${ }^{13}$ P. T. Cummings and G. Stell, Mol. Phys. 60, 1315 (1987).

${ }^{14}$ S. F. Edwards, Proc. Phys. Soc. London 85, 613 (1965).

${ }^{15}$ P. G. de Gennes, Scaling Concepts in Polymer Physics (Cornell University Press, Ithaca, NY, 1985).

${ }^{16}$ G. J. Fleer, M. A. Cohen Stuart, J. Scheutjens, T. Cosgrove, and B. Vincent, Polymers at Interfaces (Chapman and Hall, London, 1993).

${ }^{17}$ J. van der Gucht, N. A. M. Besseling, and G. J. Fleer, J. Chem. Phys. 119, 8175 (2003).

${ }^{18}$ J. van der Gucht, N. A. M. Besseling, and G. J. Fleer, Macromolecules 37, 3026 (2004).

${ }^{19}$ E. H. Feng and G. H. Fredrickson, Macromolecules 39, 2364 (2006).

${ }^{20}$ G. H. Fredrickson, The Equilibrium Theory of Inhomogeneous Polymers (Oxford University Press, New York, 2006).

${ }^{21}$ E. Guggenheim, Applications of Statistical Mechanics (Clarendon, Oxford, 1966).

${ }^{22}$ J. van der Gucht and N. A. M. Besseling, Phys. Rev. E 65, 051801 (2002).

${ }^{23}$ J. van der Gucht, N. A. M. Besseling, and M. A. Cohen Stuart, J. Am. Chem. Soc. 124, 6202 (2002).

${ }^{24}$ J. van der Gucht and N. A. M. Besseling, J. Phys.: Condens. Matter 15, 6627 (2003).

${ }^{25}$ H. J. A. Zweistra, N. A. M. Besseling, and M. A. Cohen Stuart, J. Phys. Chem. B 110, 18629 (2006).

${ }^{26}$ H. J. A. Zweistra and N. A. M. Besseling, Phys. Rev. E 74, 021806 (2006).

${ }^{27}$ M. S. Wertheim, J. Stat. Phys. 35, 19 (1984).

${ }^{28}$ M. S. Wertheim, J. Stat. Phys. 35, 35 (1984).

${ }^{29}$ M. S. Wertheim, J. Stat. Phys. 42, 459 (1986)

${ }^{30}$ M. S. Wertheim, J. Stat. Phys. 42, 477 (1986).

${ }^{31}$ M. S. Wertheim, J. Chem. Phys. 85, 2929 (1986).

${ }^{32}$ M. S. Wertheim, J. Chem. Phys. 88, 1145 (1988).

${ }^{33}$ W. G. Chapman, K. E. Gubbins, G. Jackson, and M. Radosz, Fluid Phase Equilib. 52, 31 (1989).

${ }^{34}$ E. A. Müller and K. E. Gubbins, Ind. Eng. Chem. Res. 40, 2193 (2001).

${ }^{35}$ E. Kierlik and M. L. Rosinberg, J. Chem. Phys. 97, 9222 (1992).

${ }^{36}$ E. Kierlik and M. L. Rosinberg, J. Chem. Phys. 99, 3950 (1993).

${ }^{37}$ A. Dominik, S. Tripathi, and W. G. Chapman, Ind. Eng. Chem. Res. 45, 6785 (2006).

${ }^{38}$ S. Jain, A. Dominik, and W. G. Chapman, J. Chem. Phys. 127, 244904 (2007). 
${ }^{39}$ P. Bryk, K. Bucior, S. Sokolowski, and G. Zukocinski, J. Phys.: Condens. Matter 16, 8861 (2004).

${ }^{40}$ P. Bryk, S. Sokolowski, and O. Pizio, J. Chem. Phys. 125, 204709 (2006).

${ }^{41}$ D. Cao and J. Wu, Macromolecules 38, 971 (2005).

${ }^{42}$ D. Cao and J. Wu, Langmuir 21, 9786 (2005).

${ }^{43}$ C. J. Segura, W. G. Chapman, and K. P. Shukla, Mol. Phys. 90, 759 (1997).

${ }^{44}$ C. Segura, J. Zhang, and W. Chapman, Mol. Phys. 99, 1 (2001).

${ }^{45}$ Y. Yu and J. Wu, J. Chem. Phys. 116, 7094 (2002).

${ }^{46}$ A. Bymaster, A. Dominik, and W. G. Chapman, J. Phys. Chem. C 111, 15823 (2007).

${ }^{47}$ J. Wu, AIChE J. 52, 1169 (2006).

${ }^{48}$ W. G. Chapman, K. E. Gubbins, C. G. Joslin, and C. G. Gray, Fluid Phase Equilib. 29, 337 (1986).

${ }^{49}$ D. Ghonasgi and W. G. Chapman, Mol. Phys. 79, 291 (1993).

${ }^{50}$ T. Li and E. Nies, J. Phys. Chem. B 111, 8131 (2007).

${ }^{51}$ C. J. Segura and W. G. Chapman, Mol. Phys. 86, 415 (1995).

${ }^{52}$ A. Milchev and D. P. Landau, J. Chem. Phys. 104, 9161 (1996).

${ }^{53}$ C.-C. Chen and E. E. Dormidontova, Macromolecules 39, 9528 (2006).

${ }^{54}$ A. Milchev, J. P. Wittmer, and D. P. Landau, J. Chem. Phys. 112, 1606 (2000).

${ }^{55}$ W. Knoben, N. Besseling, L. Bouteiller, and M. A. Cohen Stuart, Phys. Chem. Chem. Phys. 7, 2390 (2005).

${ }^{56}$ P. Flory, Statistical Mechanics of Chain Molecules (Interscience, New York, 1969).

${ }^{57}$ W. Knoben, N. Besseling, and M. A. Cohen Stuart, Macromolecules 39, 2643 (2006)

${ }^{58}$ W. G. Chapman, Ph.D. thesis, Cornell University, 1988.

${ }^{59}$ S. Tan, H. Adidharma, and M. Radosz, Ind. Eng. Chem. Res. 43, 203 (2004).

${ }^{60}$ G. Jackson, W. G. Chapman, and K. E. Gubbins, Mol. Phys. 65, 1 (1988).

${ }^{61}$ P. Hohenberg and W. Kohn, Phys. Rev. 136, B864 (1964).
${ }^{62}$ R. Evans, Adv. Phys. 28, 143 (1979).

${ }^{63}$ G. H. Golub and C. F. V. Loan, Matrix Computations, 3rd ed. (The Johns Hopkins University Press, Baltimore, MD, 1996).

${ }^{64}$ S. Bellavia, M. Macconi, and B. Morini, Appl. Numer. Math. 44, 257 (2003).

${ }^{65}$ S. P. Tan, H. Adidharma, and M. Radosz, Ind. Eng. Chem. Res. 47, 8063 (2008).

${ }^{66}$ Y. Rosenfeld, Phys. Rev. Lett. 63, 980 (1989).

${ }^{67}$ H. T. Davis, Statistical Mechanics of Phases, Interfaces, and Thin Films (VCH, New York, 1996).

${ }^{68}$ P. Tarazona and L. Vicente, Mol. Phys. 56, 557 (1985).

${ }^{69}$ Y. Rosenfeld, M. Schmidt, H. Löwen, and P. Tarazona, Phys. Rev. E 55, 4245 (1997)

${ }^{70}$ H. Hansen-Goos and R. Roth, J. Phys.: Condens. Matter 18, 8413 (2006).

${ }^{71}$ R. Roth, R. Evans, A. Lang, and G. Kahl, J. Phys.: Condens. Matter 14, 12063 (2002).

${ }^{72}$ J. K. Percus and G. J. Yevick, Phys. Rev. 110, 1 (1958).

${ }^{73}$ Y. Zhou and G. Stell, Int. J. Thermophys. 9, 953 (1988).

${ }^{74} \mathrm{We}$ used this expression instead of that of $\mathrm{Yu}$ and $\mathrm{Wu}$ because theirs corresponds to the BMCSL equation of state. This was done in order to avoid mixing equations of state for the different parts of the free energy.

${ }^{75}$ We thank the referees for pointing this optimization out.

${ }^{76}$ M. Michelsen and E. Hendriks, Fluid Phase Equilib. 180, 165 (2001).

${ }^{77}$ G. A. Mansoori, N. F. Carnahan, K. E. Starling, and T. W. Leland, J. Chem. Phys. 54, 1523 (1971).

${ }^{78}$ P. J. Flory, Principles of Polymer Chemistry (Cornell University Press, Ithaca, NY, 1953)

${ }^{79}$ J. P. Hansen and I. R. McDonald, Theory of Simple Liquids (Academic, London, 2006).

${ }^{80}$ J. R. Henderson and F. van Swol, Mol. Phys. 51, 991 (1984).

${ }^{81}$ W. Knoben, N. A. M. Besseling, and M. A. Cohen Stuart, Langmuir 23, 6095 (2007) 